Supportive Care and Others

\title{
Bacteriological Profile and Antibiotic Sensitivity Pattern of Clinical Isolates in a Tertiary Cancer Care Center in the Northeast India
}

\author{
Abhijit Talukdar ${ }^{1}$ Rashmisnata Barman ${ }^{2}$ Anupam Sarma ${ }^{3}$ Jagannath Dev Sharma ${ }^{3}$ \\ Manigreeva Krishnatreya ${ }^{4}$ Munlima Hazarika ${ }^{5}$ Amal Chandra Kataki ${ }^{6}$
}

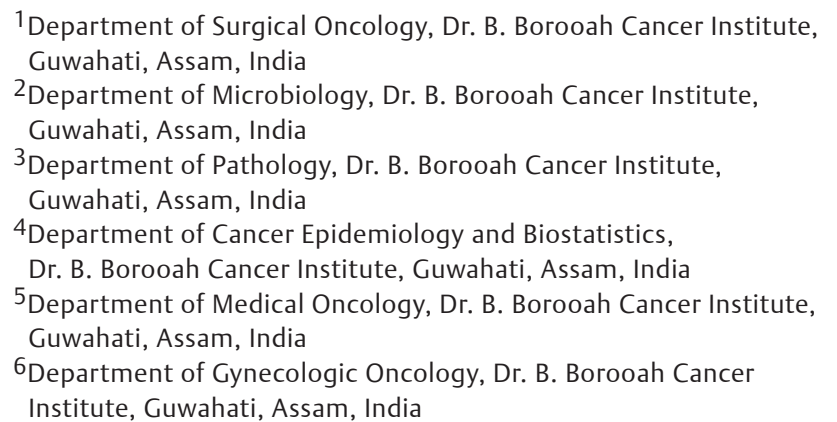

Address for correspondence Dr Rashmisnata Barman, MD Microbiology, Assistant Professor of Microbiology, Department of Microbiology, Dr. B. Borooah Cancer Institute, Guwahati, Assam, India (e-mail: drrashmisnata@gmail.com).

\begin{abstract}
Keywords

- extended-spectrum $\beta$-lactamase

- immunocompromised

- methicillin-resistant

Staphylococcus aureus

- multidrug resistance
\end{abstract}

Background This study was performed to determine the bacteriological profile and antibiotic sensitivity pattern of culture samples of patients with cancer at our institute. The study was undertaken to formulate an antibiotic policy for the treatment of infection in these patients.

Materials and Methods The study was performed in the Department of Microbiology of a regional cancer center during the period from January 2017 to December 2017. Samples were collected under all aseptic precaution, and they were processed as per the Clinical and Laboratory Standard Institute Guideline 2017.

Results A total of 464 clinical samples (urine, blood, sputum, pus, etc.) were collected and processed for culture, of which 198 (42.67\%) samples showed culture positive that were identified as per standard recommended procedures and antibiotic susceptibility testing was performed on isolates as per the Clinical Laboratory Standard Institute guidelines 2017. Escherichia coli (48), Staphylococcus aureus, (45) Klebsiella pneumoniae (52), Coagulase-negative Staphylococcus (17), and Pseudomonas aeruginosa (15) were most commonly encountered. Of the $132 \mathrm{Gram}$-negative isolates, 101 (76.5\%) were extended-spectrum $\beta$-lactamase producers. Among the 45 staphylococcal isolates, 18 (40\%) were methicillin-resistant S. aureus.

Conclusion The present study reveals microbiological profile in patients attending our cancer institute.
DOI https://doi.org/ $10.1055 / \mathrm{s}-0040-1721176$ ISSN 2278-330X. (c) 2020. Medlntel Services Pvt Ltd.

This is an open access article published by Thieme under the terms of the Creative Commons Attribution-NonDerivative-NonCommercial-License, permitting copying and reproduction so long as the original work is given appropriate credit. Contents may not be used for commercial purposes, or adapted, remixed, transformed or built upon. (https://creativecommons.org/licenses/by-nc-nd/4.0/) Thieme Medical and Scientific Publishers Pvt. Ltd., A-12, 2nd Floor Sector 2, Noida-201301 UP, India 


\section{Introduction}

Cancer increases a patient's risk of getting a serious infection. In spite of the recent advances made by medical science in cancer treatment, infections still remain a major cause of morbidity and mortality in patients diagnosed with cancer. ${ }^{1,2}$ Cancer patients are often immunocompromised because of the disease process itself and also due to various interventions such as chemotherapy. In addition, there are usually other associated risk factors for acquiring infection such as long-term catheterization, mucositis due to cytotoxic agents, neutropenia, and stem-cell transplantation. ${ }^{3}$ Infectious complications are a serious cause of morbidity and mortality in patients with underlying hematological malignancies. Moreover, in solid tumors, although there is no prolonged neutropenia, the presence of multiple other risk factors is responsible for an immunocompromised state, like there is obstruction caused by the tumor, disruption of normal anatomical barriers, therapeutic procedures, chemotherapy, radiation, and use of medical devices such as catheter, stent, and prosthesis. 4,5 The common sites of infection seen in cancer patients undergoing treatment are bloodstream, respiratory, gastrointestinal tract, skin soft tissue, and urinary tract infections. In the present-day context, there is the emergence of multidrug-resistant organisms in certain infections. Antimicrobial resistance poses a major threat to patient's treatment, as it leads to severe morbidity and high-mortality rates, increased hospital stay, and severe economic burden to the patient and healthcare system. ${ }^{4}$

Empirical therapy with broad-spectrum antibiotic and subsequently de-escalating to a narrower-spectrum drug after culture sensitivity results are available is the current approach for the treatment of cancer patients with infection. This study aimed to document the most common bacterial profile and the antimicrobial susceptibility patterns in clinical samples of cancer patients undergoing treatment at our center.

\section{Materials and Methods}

Two hundred and fifty patients with various malignancies were included in this study. The present study was conducted for 1 year in a Regional Cancer Center in the Northeast India. This was a retrospective and observational hospital-based study performed at the Department of Microbiology of the institute. The Institutional Ethical Committee's clearance was obtained prior to conducting the study. This study was conducted on all clinical isolates from samples of patients received from different oncology units from January 2017 to December 2017. All relevant samples were collected as per hospital sample collection protocol from various clinical areas; these included urine, skin and soft tissue, blood, and respiratory samples. The clinical data were obtained from the requisition forms and from the respective units and wards of the patient. All samples were processed as per standard microbiology laboratory standard operating procedures. ${ }^{6,7}$ The isolates were identified by their colonial morphology, Gramstaining, and different biochemical reactions using standard techniques. Criteria for antimicrobial sensitivity testing were performed as per the Clinical Laboratory Standard Institute (CLSI, United States) guidelines. ${ }^{8}$ Antimicrobial sensitivity testing was done on Mueller-Hinton agar by KirbyBauer's disc diffusion method. Commercially available discs (HiMedia) were used. Zones of inhibition were measured the next day and were correlated with CLSI interpretive breakpoints to characterize them as sensitive, intermediate, and resistant. For drugs for which CLSI breakpoints were not available, interpretative breakpoints were provided by the manufacturer. Staphylococcus aureus ATCC 25923, Escherichia coli ATCC 25922, and Pseudomonas aeruginosa ATCC 27853 were used for quality control.

For Gram-positive organisms, the antibiotics to be tested and reported were chosen from the following (depending on the organism isolated): Penicillin, erythromycin, clindamycin, gentamicin, and high level, amoxicillin-clavulanate, cefoxitin, levofloxacin, vancomycin linezolid, and cotrimoxazole.

For Gram-negative, the antibiotics for respective organisms were chosen from the following: amoxicillin-clavulanate, ciprofloxacin, levofloxacin, gentamicin, amikacin, netilmicin, cefuroxime, cefotaxime, ceftazidime, cefepime, cefoperazone-sulbactam, cefepime-tazobactam, imipenem, and meropenem. Colistin susceptibility was performed using minimum inhibitory concentration method, with E-test strips. In the present study, vancomycin susceptibility test could not be done by microbroth dilution method as recommended, due to nonavailability of this facility in the institute where this study was conducted.

\section{Results}

A total of 464 samples of 250 patients were received and processed from different departments of the institute as shown in - Table 1. Of the 250 patients, 108 were $<40$ years of age and 142 were above 40 years of age. The male-to-female ratio

Table 1 Samples received and processed from different departments of the institute

\begin{tabular}{|l|l|l|l|l|}
\hline \multirow{2}{*}{ Specimen } & \multicolumn{4}{|c|}{ Specimen received for bacterial culture } \\
\cline { 2 - 5 } & Medical oncology & Surgical oncology & Gynecologic oncology & Head and neck \\
\hline Urine & 30 & 34 & 19 & 3 \\
\hline Blood & 240 & 6 & 0 & 0 \\
\hline Respiratory & 15 & 9 & 4 & 28 \\
\hline Skin and soft tissue & 10 & 28 & 14 & 24 \\
\hline Total & 295 & 77 & 37 & 55 \\
\hline
\end{tabular}


Table 2 Patient profiles of the present study

\begin{tabular}{|l|l|}
\hline Patient profile & $\boldsymbol{n}$ \\
\hline Total number of patients & 250 \\
\hline Age (y) & \\
\hline$<40$ & 108 \\
\hline$>40$ & 142 \\
\hline Male & 166 \\
\hline Female & 84 \\
\hline Type of malignancies & \\
\hline Hematological malignancies & 104 \\
\hline Gastrointestinal malignancies & 69 \\
\hline Gynecological malignancies & 31 \\
\hline Head-and-neck malignancies & 46 \\
\hline Patients with neutropenia & 130 \\
\hline Patients without neutropenia & 120 \\
\hline
\end{tabular}

Table 3 Total number of culture positive of each specimen

\begin{tabular}{|l|l|l|}
\hline Specimen & Total & Culture positive (\%) \\
\hline Urine & 86 & $64(74)$ \\
\hline Respiratory & 56 & $40(71)$ \\
\hline Skin and soft & 76 & $32(42)$ \\
\hline Blood & 246 & $62(25)$ \\
\hline
\end{tabular}

was 2.1. Type of malignancies detected in the present study were hematological malignancies in 104 (41.6\%), gastrointestinal malignancies in 69 (27.6\%), gynecological malignancies in 31 (12.4\%), and head-and-neck malignancies in $46(18.4 \%)$ patients. One hundred and thirty $(52 \%)$ patients were neutropenic and 120 (48\%) patients were nonneutropenic (-Table 2). The highest number of neutropenic patients was cases with hematological malignancies followed by gastrointestinal malignancies and gynecological malignancies. Neutropenic patients were mainly in the age group of $<40$ years. Of 130 neutropenic patients, blood culture was positive in 46 patients.

One-hundred and ninety-eight (42.67\%) organisms were isolated. The total number of organisms isolated from various clinical samples is shown in - Table 3.

Of the 132 Gram-negative isolates, 101 (76.51\%) were extended-spectrum $\beta$-lactamase (ESBL) producers as shown in - Table 3. Carbapenem resistance in E. coli and Klebsiella pneumoniae was $18 \%$ and $19 \%$, respectively. Of the 45 staphylococcal isolates, 18 (40\%) were methicillin-resistant S. aureus (MRSA) (- Table 4).

All the $S$. aureus isolates were sensitive to vancomycin (100\%), linezolid (100\%), and teicoplanin (100) (-Fig. 1A).

In the present study, sensitivity pattern of E. coli was to colistin (100\%), imipenem (82\%), meropenem (88\%), ertapenem (80\%), piperacillin-tazobactam (68\%), amikacin (84\%), netilmicin (89\%), gentamicin (92\%), and cefepime (46\%) (-Fig. 1B).

In the present study, sensitivity pattern of the K. pneumoniae was to colistin (100\%), imipenem (81\%), meropenem (82\%), ertapenem (80\%), piperacillin-tazobactam (67\%), amikacin (89\%), netilmicin (92\%), gentamicin (68\%) and cefepime (32\%) (-Fig. 1C).

In the present study, sensitivity pattern of the P. aeruginosa was to colistin (100\%), imipenem (84\%), meropenem (86\%), piperacillin-tazobactam (76\%), amikacin (82\%), netilmicin (94\%), gentamicin (85\%), and cefepime (57\%) (-Fig. 1D).

\section{Discussion}

The type of malignancy, the status of the malignancy (i.e., active or remission), and the intensity of the treatment directed against it are all important factors in determining infection risk. ${ }^{9}$ In cancer patients, there is increased risk of skin and soft-tissue infection and bacteremia. ${ }^{9}$ In this study, patient specimen showed a higher number of urinary tract infection followed by skin and soft tissue, respiratory, and blood. However, some of these isolates from urinary and respiratory trace could be colonizers and this study was not powered to differentiate between the two.

Among the Gram-negative isolates, there were high rates of resistance to the third-generation cephalosporins (cefotaxime/ceftazidime) and also to the $\beta$-lactam- $\beta$-lactamase inhibitor combinations such as cefoperazone-sulbactam and piperacillin-tazobactam. Among the 62 Gram-positive Staphylococcal isolates, 18 (29\%) were MRSA. Among the $S$. aureus isolates, all were sensitive to vancomycin, linezolid, and teicoplanin.

Enterococcus faecalis was isolated from four urine samples of gynecology oncology. Gynecological malignancy disrupts barriers in the female genitourinary tract predisposing to infection with Enterococci, enteric aerobes, and anerobic bacteria. ${ }^{9}$

In our study, out of 246 blood cultures, 62 were positive. Patients with neoplasms presenting with Enterobacteriaceae bacteremia were more likely to have infection with $K$. pneumoniae and E. coli. This association may be explained by host and pathogen factors. ${ }^{10}$ The culture isolates were K. pneumoniae (26), E. coli (22), and S. aureus (14). All Enterobacteriaceae isolates were ESBL producer. Moreover, this is a real matter of great concern.

Out of the total 132 Gram-negative isolates from all clinical specimen, 101 (76.51\%) were ESBL producers. Carbapenem resistance in E. coli and K. pneumoniae was 18\% and $19 \%$, respectively. Similar finding with rates of multidrug resistance organisms has been noted in a study from Mumbai. ${ }^{1,2}$ However, fortunately, the antimicrobial profile of carbapenems (imipenem, meropenem, ertapenem) and aminoglycosides (gentamicin, amikacin, netilmicin) showed $>50 \%$ sensitivity. Although a susceptibility of $50 \%$ is not enough for empirical treatment, for nonneutropenic patients, carbapenems can be an empirical choice of treatment. Aminoglycosides are to be preserved for use in the urinary tract as they achieve good concentration there.

Moreover, all Gram-negative isolated were sensitive to colistin. Similar results were seen in a study conducted in a tertiary care cancer center in Delhi. ${ }^{1}$ Carbapenems-resistant 
Table 4 Organisms isolated from different departments

\begin{tabular}{|c|c|c|c|c|c|c|c|}
\hline Organisms isolated & $\begin{array}{l}\text { Medical } \\
\text { oncology }\end{array}$ & $\begin{array}{l}\text { Surgical } \\
\text { oncology }\end{array}$ & $\begin{array}{l}\text { Gynecology } \\
\text { oncology }\end{array}$ & $\begin{array}{l}\text { Head } \\
\text { and neck } \\
\text { oncology }\end{array}$ & Total & MRSA & ESBL \\
\hline Escherichia coli & 34 & 6 & 8 & 0 & 48 & & 40 \\
\hline Klebsiella pneumoniae & 30 & 10 & 2 & 10 & 52 & & 43 \\
\hline Staphylococcus aureus & 17 & 17 & 7 & 4 & 45 & 12 & \\
\hline $\begin{array}{l}\text { Coagulase negative } \\
\text { staphylococcus }\end{array}$ & 5 & 6 & 4 & 2 & 17 & 6 & \\
\hline Pseudomonas aeruginosa & 3 & 4 & 3 & 5 & 15 & & 7 \\
\hline Serratia odorifera & 0 & 0 & 1 & 0 & 1 & & 1 \\
\hline Citrobacter spp. & 0 & 1 & 2 & 0 & 3 & & 2 \\
\hline Proteus vulgaris & 2 & 3 & 2 & 0 & 7 & & 4 \\
\hline Proteus mirabilis & 3 & 1 & 0 & 0 & 4 & & 3 \\
\hline Morganella morganii & 1 & 0 & & 0 & 1 & & 1 \\
\hline Serratia marcescens & 0 & 1 & 0 & 0 & 1 & & 0 \\
\hline Enterococcus faecalis & & & 4 & & 4 & & \\
\hline
\end{tabular}

Abbreviations: ESBL, extended spectrum $\beta$ lactamase; MRSA, methicillin-resistant Staphylococcus aureus.
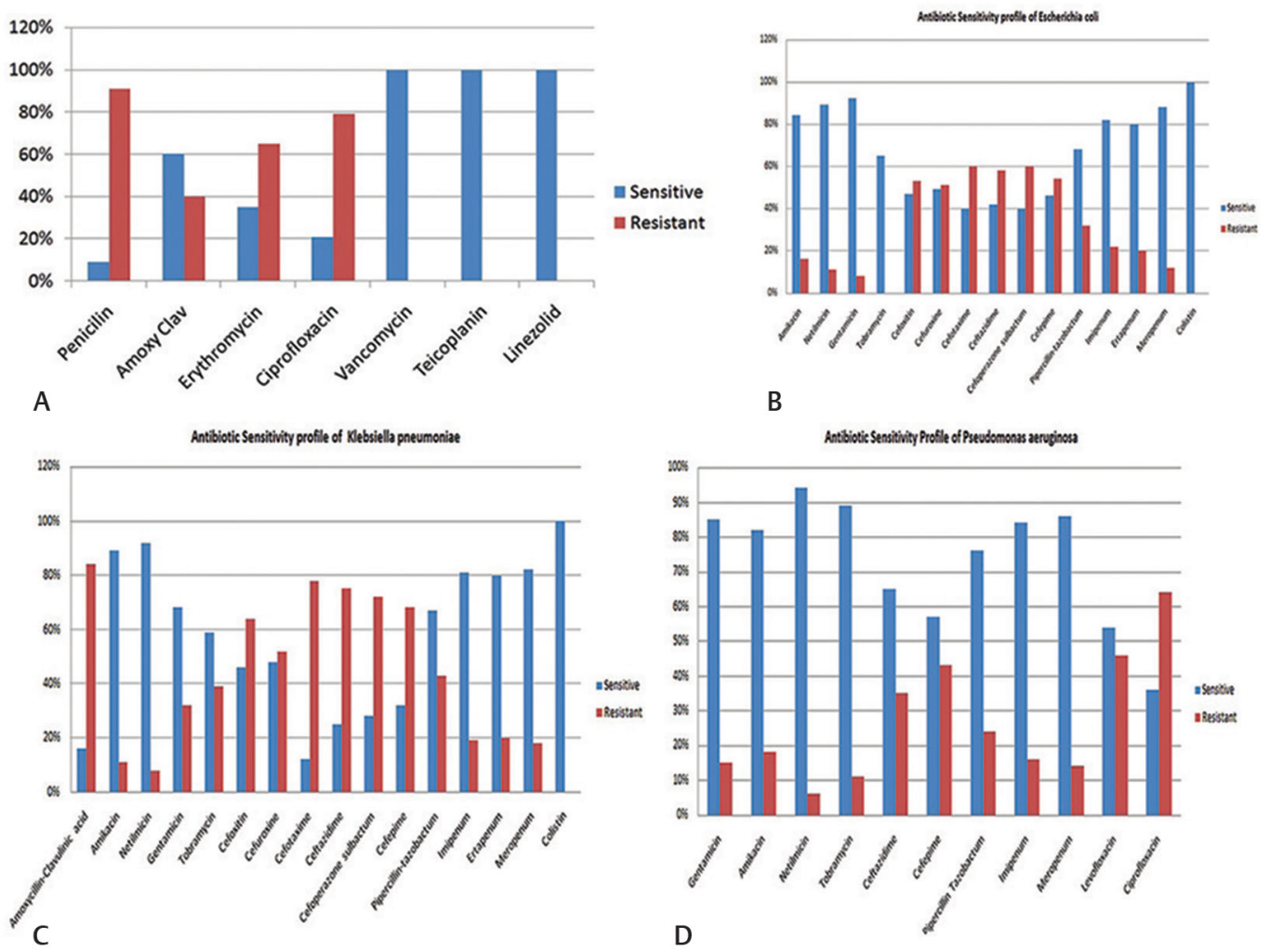

Fig. 1 The sensitivity profile of (A) Staphylococcus aureus. (B) Escherichia coli, (C) Klebsiella pneumoniae, and (D) Pseudomonas.

Enterobacteriaceae group of organisms, particularly E. coli and K. pneumoniae, is a worrying factor.

Antibiotic resistance is now emerging among the Gram-positive isolates. High rates of methicillin resistance ranging from 54 to $72.3 \%$ and the emergence of vancomycin-intermediate strains of $S$. aureus have been reported from India. ${ }^{11,12}$ We did not encounter any vancomycin resistance among staphylococci, and MRSA rates have been approximately $34.28 \%$ in another study, ${ }^{12}$ and in the present study, MRSA was $40 \%$. 
This study showed that the most common organism present in our clinical samples was Gram-negative aerobes. S. aureus was the single most common predominant organism isolated from various specimens. The presence of multidrug-resistant organisms was alarmingly high, especially from blood culture isolates. These observations are important, especially for the management and development of empirical antibiotic guidelines. In the present healthcare scenario, it is very important for every healthcare setting to formulate antibiotic policies based on local antibiotic susceptibility patterns so that arbitrary use of antibiotics is avoided and resistance is kept to a minimum.

Neutropenia is a known factor influencing mortality and morbidity. ${ }^{13}$ This may be because only cases with severe infections were sent for blood culture. Bacteremia was present in 46 out of 130 samples, but the association of multidrug-resistant strains from these isolates was an important factor. Neutropenic patients were mainly in the $<40$ years age group. In our study, bacteremia was seen in $35 \%$ of the patients compared with $38 \%$ as observed by Hurtado et al. ${ }^{14}$

\section{Limitations of the Study}

There are several limitations to the study. Mainly, we used manual (Kirby-Bauer's) disc diffusion method for sensitivity analysis. Furthermore, the present study did not examine for anaerobic isolates and fungal isolates in cancer patients undergoing treatment. Furthermore, the present study was not powered to differentiate colonizers from pathogens. Colistin and vancomycin susceptibility could not be done by broth microdilution method.

\section{Conclusion}

We emphasize the regular evaluation of local isolates and sensitivity pattern in cancer patients undergoing treatment. In our experience, in cancer patients undergoing a various form of treatment, carbapenem along with aminoglycosides such as netilmicin and tobramycin are standard options for Gram-negative coverage, and linezolid, teicoplanin, and vancomycin can be used for treating Gram-positive infection in cancer patients. It was seen that isolates from bloodstream infections in neutropenic patients were predominantly multidrug resistance organism. It is recommended that every institution should regularly revise their antimicrobial policy based on microbiological data.

\section{Funding}

Nil.

\section{Conflict of Interest}

There are no conflicts of interest.

\section{References}

1 Singh R, Jain S, Chabbra R, Naithani R, Upadhyay A, Walia M. Characterization and anti-microbial susceptibility of bacterial isolates: experience from a tertiary care cancer center in Delhi. Indian J Cancer 2014;51(4):477-480

2 Bhat V, Gupta S, Kelkar R, et al. Bacteriological profile and antibiotic susceptibility patterns of clinical isolates in a tertiary care cancer center. Indian J Med Paediatr Oncol 2016;37(1):20-24

3 Hughes WT, Armstrong D, Bodey GP, et al; Infectious Diseases Society of America. 2002 guidelines for the use of antimicrobial agents in neutropenic patients with cancer. Clin Infect Dis 2002;34(6):730-751

4 Nazneen S, Mukta K, Santosh C, Borde A. Bacteriological trends and antibiotic susceptibility patterns of clinical isolates at Government Cancer Hospital, Marathwada. Indian J Cancer 2016;53(4):583-586

5 Rolston KV. Infections in cancer patients with solid tumors: a review. Infect Dis Ther 2017;6(1):69-83

6 Koneman EW, Allen SD, Janda WM, Schreckember PC, Winn WC. Koneman's Colour Atlas and Textbook of Diagnostic Microbiology. 6th edition. New York: Lippincott; 2006:97-99

7 Forbes BA, Sahm DF, Weissfeld AS, Bailey and Scott's Diagnostic Microbiology. 12th edition. Missouri: Mosby Elsevier; 2007:779

8 Clinical and Laboratory Standards Institute, Clinical and Laboratory Standards Institute 2016. Performance Standards for Antimicrobial Susceptibility Testing. Twenty-Second Informational Supplement. Wayne, PA: Clinical and Laboratory Standards Institute; 2016

9 Zembower TR. Epidemiology of infections in cancer patients. Cancer Treat Res 2014;161:43-89

10 Henao-Martínez AF, González-Fontal GR, Castillo-Mancilla JR, Yang IV. Enterobacteriaceae bacteremias among cancer patients: an observational cohort study. Int J Infect Dis 2013;17(6):e374-e378

11 Menezes GA, Harish BN, Sujatha S, Vinothini K, Parija SC. Emergence of vancomycin-intermediate Staphylococcus species in southern India. J Med Microbiol 2008;57(Pt 7) :911-912

12 Eshwara VK, Munim F, Tellapragada C, et al. Staphylococcus aureus bacteremia in an Indian tertiary care hospital: observational study on clinical epidemiology, resistance characteristics, and carriage of the Panton-Valentine leukocidin gene. Int J Infect Dis 2013;17(11):e1051-e1055

13 Ghosh I, Raina V, Kumar L, et al. Profile of infections and outcome in high-risk febrile neutropenia: experience from a tertiary care cancer center in India. Med Oncol 2012;29(2):1354-1360

14 Hurtado IC, Sánchez DP, Espinal DA, Garcés C. [Clinical and laboratory evolution of the febrile neutropenia episodes in pediatric patients hospitalized in a Colombian hospital in 2007-2009]. Rev Chilena Infectol 2012;29(6):672-676 